\title{
Managing the water distribution network with a Smart Water Grid
}

\author{
Public Utilities Board Singapore
}

\author{
* Correspondence: \\ Pan_Ju_Khuan@pub.gov.sg \\ Public Utilities Board, 40 Scotts \\ Road \#10-01 Environment Building \\ S(228231), Singapore, Singapore
}

\begin{abstract}
In Singapore, the implementation of a Smart Water Grid system supports the Public Utilities Board (PUB)'s mission to supply good water 24/7 to its customers. With sensors and analytic tools deployed island-wide to provide a real-time monitoring and decision support system, the Smart Water Grid system enables PUB to manage the water supply network efficiently, ensuring that all Singaporeans will continue to enjoy a reliable and sustainable water supply for generations to come. This paper covers the five key aspects of the Smart Water Grid system, in relation to both the key operational aspects of a water distribution system (asset management, leak management, water quality monitoring) and the customers' end (automated meter reading and water conservation). For each aspect, the paper focuses on the challenges, current technology, PUB's experience with technology and identified gaps, and the technology roadmap.

Keywords: Smart Water Grid, Water management, Water infrastructure, Smart technology, ICT, Wireless sensor network, PUB, Singapore
\end{abstract}

\section{Introduction}

\section{Context \& challenges}

PUB currently supplies 430 millions of gallons per day (MGD) of water to customers in Singapore. The Water Supply Network (WSN) Department manages the $5490 \mathrm{~km}$ of potable mains, $573 \mathrm{~km}$ of NEWater (high-grade reclaimed water) mains and $42 \mathrm{~km}$ of industrial water mains that deliver the water to more than 1.4 million customers. WSN has embarked on a Smart Water Grid journey to ensure good water is supplied to its customers $24 \mathrm{~h}$ a day, 7 days a week. A Smart Water Grid system integrates information and communications technologies (ICT) into the management of the water distribution system. Sensors, meters, digital controls and analytic tools are used to automate, monitor and control the transmission and distribution of water, ensuring that water is efficiently delivered only when and where it is needed and with good quality. Today, many industries are making use of ICT to improve their operations and processes, particularly through digitization and automation. Digitization and automation enable automatic remote collection of data at site, and wireless transmission to a central system for monitoring and analysis. Recent advancements in ICT have been largely driven by the emergence of Internet of Things, cloud computing and big data analytics. When applied to the water industry, these advances in ICT enable to capture and store enormous pools of big data and perform powerful analytics and predictive analysis to contribute towards greater efficiency and effectiveness in water resource management. As the Singapore population

(C) 2016 Public Utilities Board Singapore. Open Access This article is distributed under the terms of the Creative Commons Attribution 4.0 International License (http://creativecommons.org/licenses/by/4.0/), which permits unrestricted use, distribution, and reproduction in any medium, provided you give appropriate credit to the original author(s) and the source, provide a link to the Creative Commons license, and indicate if changes were made. 
continues to grow, PUB's water infrastructure needs to be ready to meet an increasing water demand while maintaining good asset condition to ensure good water is supplied to customers around the clock. There is an increasing need to leverage on these innovative technologies in order to ensure the water infrastructure is sustainable and resilient. Presently in WSN, many of the operational processes such as water leakage checks, water quality checks and meter readings, are still done manually. Against the backdrop of a shrinking and increasingly educated labour force, the implementation of the Smart Water Grid will also streamline manpower requirements and redesign work processes by automating menial tasks.

The expected benefits of a Smart Water Grid are the following:

- Real-time monitoring of asset condition for preventive maintenance. With advanced sensing technologies, data on pipeline condition can be used to develop a risk-based model for pipe replacement projects. This enables WSN to better plan and schedule the mains replacement and rehabilitation programme, so that the right pipes are replaced at the right time.

- Real-time pressure and water quality monitoring to enhance planning and network operations. Real-time sensor and meter data allow WSN to monitor the hydraulic and water quality situation throughout the network. Leaks can be located promptly to minimize water losses, and stress in pipes can be detected early so that actions can be taken to mitigate the risk of pipe bursts. Continuous monitoring of the water quality in the distribution pipelines also provides early warning of potential contamination. Automated valve operations can then respond by shutting valves in the affected areas to prevent flooding, further damages, water loss, or spreading of the contaminated water. Real time sensor data can also be used for calibration of hydraulic models, to replace the traditional calibration process which is tedious, time-consuming and expensive.

- Real-time water consumption information to help customers conserve water. On the customers' end, WSN hopes to use technologies like automated meter readings and smart water efficient gadgets provide real-time feedback on water and energy usage to customers. This enables customers to make informed choices towards water conservation within their homes and company premises. In addition, the usage data from automated meter readings will enable more accurate demand prediction for optimizing pumping schedules, water turn-over in service reservoirs, and water required to be treated and pumped.

Decision support tools, such as predictive hydraulic modelling, valve operation simulation, demand prediction modelling and pipeline failure analysis, help to improve network assessments and planning.

Through trials of various Smart Water Grid technologies, WSN has identified a few key challenges. Firstly, there is a lack of interoperability technical standards for the three primary components of the Smart Water Grid, namely sensors, wireless communications and data analytics tools. This impedes the integration of multiple components of the Smart Water Grid system. Secondly, real-time sensor and meter readings generate massive amounts of data which require good organization and powerful analytics in order to extract useful information. Thirdly, job-redesign for existing staff must be considered 
as new roles are created and old roles made redundant with the implementation of Smart Water Grid. Fourth, public communications must address concerns adequately to ensure that society is accepting of the technology. Finally, as Smart Water Grid technologies have yet to mature, further research and testing will be needed to realize the full benefits of a Smart Water Grid.

This paper covers the five key aspects of the Smart Water Grid system, in relation to both the key operational aspects of a water distribution system (asset management, leak management, water quality monitoring) and the customers' end (automated meter reading and water conservation). For each aspect, the paper focuses on the challenges, current technology, PUB's experience with technology and identified gaps, and the technology roadmap.

\section{Main text}

\section{Asset management}

Objectives \& challenges

Most of WSN's pipelines are approximately 20-30 years of age, with an expected asset lifespan of 70 years. As WSN continues to lay more pipelines, the asset base grows, which translates to more potential points of failure. WSN is adopting a proactive and systematic approach to manage the asset base in order to optimize the lifespan of existing pipelines and pre-empt failures of critical pipelines. To facilitate the implementation of the new asset management system, WSN will leverage widely on condition assessment technologies to retrieve more dynamic and real time data. The challenge is that pipelines are buried, lined \& have undulating profiles. In addition, the network is interconnected \& "live" 24/7 reliable supply is required. This makes it difficult to implement condition assessment technologies on a large scale. However, once these challenges are surmounted, these data can be uploaded into an asset management software to improve the identification of high-risk pipelines so that pipeline renewal works can be prioritized. With a better understanding of the circumstances leading to pipe failures, WSN can also improve pipeline designs to prevent future failures. Leveraging on a Smart Water Grid system for asset management thus allows us to plan, design, operate and manage assets cost-effectively for safe and reliable supply to customers.

\section{Review of current technology \& tools in the market}

A risk management software system for prioritizing pipeline maintenance/replacement typically computes a risk score aggregate for each asset based on Likelihood of Failure (LOF) and Consequence of Failure (COF). The LOF of an asset is determined by multiple factors such as age of pipe, material of pipe, pressure and soil type which affect the probability of failure. COF Factors refer to elements such as sensitivity of customers and loss of pipe pressure during pipe burst. There is a wide range of both direct and indirect condition assessment techniques to determine the existing condition of a pipeline and the rate of its deterioration. Direct techniques consist of visual inspections, CCTV surveys and wall thickness measurements using remote/near field techniques, broadband electromagnetic surveys, or ultrasonic techniques. However, these direct techniques require access to either the internal or external surface of the pipe. A shutdown is usually required to gain access to the inside of a pipe, while access to the outside of the pipe typically requires excavation of the surrounding ground. Alternatively, indirect techniques can provide useful data at a lower cost, but requires 
careful interpretation by experts. These indirect techniques can include coating defect surveys, stray current surveys, soil studies, and statistical analysis of operation performance data (e.g. pressure, soil texture, age and traffic loads). WSN intends to test-bed new technologies, which are able to perform both acoustic and video inspections for wall thickness assessment without requiring shutdown.

\section{PUB experience with technology \& gaps identified}

PUB has worked with Aqleo (previously a spin-off from the National University of Singapore) to develop a Pipeline Failure Analysis Model to predict hotspots of pipe failures through an R\&D project. Using Bayesian statistics, the model is able to identify $25 \%$ of the total pipes in the network where failures are most likely to occur (Pradhan et al. 2014). WSN has also started to implement a risk management software system for its assets. However, these risk-based models can only be effective if the input data is accurate and sufficient for analysis. Most of the available condition assessment technologies are intrusive and require pipeline operations to be disrupted. This means that condition assessment can only be applied at a small number of selected sites. Methods are then needed to extrapolate the limited data over the entire length of the pipeline, and over time so that the probability of failure can be predicted and the remaining lifetime can be estimated. Even with indirect techniques, data has to be carefully analysed \& interpreted in order to provide useful information. Thus, it is necessary to develop a framework for the collection, analysis and management of useful pipeline asset data.

\section{Technology roadmap}

In order to collect more information on pipeline assets, WSN intends to collaborate with laboratories to conduct destructive testing of exposed corroded pipes, so as to understand the condition of similar pipelines in the vicinity. A database of soil data will also be built to develop a corrosion model that takes soil corrosiveness into account when determining pipeline conditions. Furthermore, WSN plans to install fibre-optic sensors along critical stretches of proposed pipelines so as to develop the capability for real time monitoring of pipeline conditions by 2020. With the incorporation of riskbased, failure forecasting models into the asset management system, WSN targets to annually identify the top $2 \%$ high risk pipes for replacement by 2020 .

\section{Leak management and enhancing network operations \\ Objectives \& challenges}

Under WSN's dynamic leak detection programme, field crew is dispatched to survey water mains for leaks. They rely on visual cues, listening sticks and leak noise localisers to identify leaks. With an extensive network of around $6000 \mathrm{~km}$ of mains, each survey cycle can take up to a year to complete. However, with increasing public expectations, there is a need to find methods to further reduce response time towards leak incidents. WSN aims to deploy more advanced leak detection equipment/systems to continuously monitor and analyse leaks, thereby improving response time and minimizing impact to customers. With more leak monitoring systems installed island-wide, there can be significant reduction in the manpower which is normally required to perform the routine site inspection. 
Review of current technology \& tools in the market Acoustic sensors based on accelerometers

Leak noise localisers and leak noise correlators are listening devices which use accelerometers to sense leak-induced sounds or vibrations caused by water escaping from a pressurized pipe. These devices are suited for the network in Singapore as metal pipes easily transmit water leak sounds (Hamilton \& Charalambous 2013). Leak noise correlators are more sensitive than localisers, as they are able to automatically pinpoint leak locations based on parameters like pipe size, distance and pipe material. However, due to the sound propagation theory, these methods are only accurate for small diameter water mains; preferably $300 \mathrm{~mm}$ mains and below. In addition, the range of the sensors is limited to $250 \mathrm{~m}$.

\section{Acoustic sensors based on hydrophone sensors}

It is also possible to determine leak positions by analysing data from hydrophone sensors that capture sound waves in water. Hydrophones are more sensitive than accelerometers and are effective in detecting leaks in larger pipes. However, such systems are known to be expensive and a detailed site study has to be conducted as the sensors are best installed in $100 \mathrm{~mm}$ or $150 \mathrm{~mm}$ air valves located not more than $750 \mathrm{~m}$ apart (Hamilton \& Charalambous 2013).

\section{High rate pressure sensors}

High Rate Sampling Pressure Sensors are used to detect patterns of pressure transients which occur during a pipe burst. These sensors have a much wider detection range of $1.5 \mathrm{~km}$, but produce lower resolution results in a range of a few hundred meters, compared to tens of metres for acoustic devices. Moreover, there is a need to distinguish pressure transients caused by leaks against those caused by network operations or regular draw-offs.

\section{Virtual district metering areas}

Virtual District Metering Areas utilize flow meters to monitor inflows and outflows to sub-sections of the water network. Combined with automated meter readings from customers premises, mass balance calculations can be used to derive the actual water loss in an area. Operators can then focus their leak survey efforts on areas where high water losses are observed.

\section{Statistical analysis of pressure and flow}

Statistical analysis of flows and pressures can be carried out to reveal deviations that may indicate a new leak. However, water flow fluctuations can be attributed to a variety of reasons such as seasonal variations and public holidays amongst other causes. Thus, statistical analysis also needs to take other datasets into account to improve its accuracy.

\section{PUB experience with technology \& gaps identified}

Leak noise localisers and correlators have already been incorporated within the Standard Operating Procedures of WSN's Leak Detection Unit. High rate pressure sensors have been installed in the network. The dedicated WaterWise software system 
developed by PUB and Visenti (http://www.visenti.com/) comprises 300 multi-parameter probes to detect both leaks and water quality issues in real-time (see Fig. 1a and b) (Allen et al. 2011; Whittle et al. 2011). Advanced computer algorithms are used to identify the pressure drop signatures associated with pipe bursts. After identifying a credible signature, the system attempts to identify the stretches of water mains which may contain the leak spot. Upon receiving the electronic alerts, officers will proceed to survey these mains for leaks. The survey efforts are therefore more focused compared with the traditional leak detection programme.

WSN has also installed a separate system of short range sensors (acoustic range: $250 \mathrm{~m}$ ) at leak-prone areas in the network. These sensors provide a higher resolution for leak localisation, but are only semi-real-time as they are switched on in the early morning when ambient noise levels are lower.

\section{Technology roadmap}

WSN plans to expand the sensor coverage in the network to $90 \%$ by 2018 with 400 additional multi-parameter sensors. The focus is now to distinguish the sharp pressure transients of leaks from the transients created by high customer consumption. WSN will also be testing the use of hydrophones to detect leaks in large transmission mains. As more trials are conducted, WSN can work with vendors to develop advanced software filters to better identify leaks.

\section{Water quality monitoring}

\section{Objectives \& challenges}

WSN's long-term goal is to monitor and model network water quality in real-time, so as to detect contamination early and control its spread to minimise impact to customers. There is a need to move away from depending on customers to act as sensors for water quality issues like discoloured water. Furthermore, in today's volatile socio-political climate, we need to be even more vigilant to deter and prevent acts of sabotage that may threaten the quality of the water supply.

\section{Review of current technology \& tools in the market Water quality sensors}

A smart sensor array in a potable water distribution network consists of live water quality sensors placed in strategic locations of the network. Several real-time water

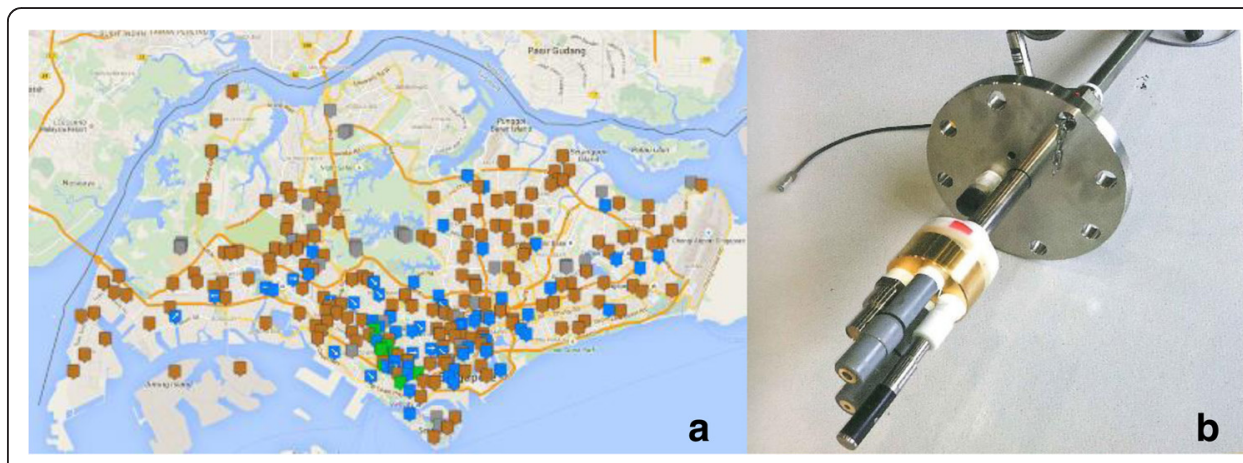

Fig. 1 a Map of WaterWise sensors and $\mathbf{b}$ image of a sensor probe 
quality monitoring sensors currently exist in the market. There are conventional sensors that directly measure specific water quality parameters such as $\mathrm{pH}$, turbidity and conductivity. There are also sensors which aim to detect any deviations in generic properties of water (such as optics) in order to cover a broader spectrum of contaminants. Finally, there are biosensors which monitor the behaviour of living organisms in the water to assess the toxicity of water samples.

\section{Water quality data analytics}

Event Detection Systems are used to analyse sensor data in real time and to alert the operators when the water quality is deemed anomalous. PUB has collaborated with Sandia National Laboratories (http://www.sandia.gov/) to develop CANARY (EPA 2010), a water quality event detection tool, in the distribution network. Several change detection algorithms are included within CANARY: a linear filter, a multivariate nearest-neighbour algorithm, and a set-point proximity algorithm. These algorithms identify a background water quality signature for each sensor and compare each new measurement to that background to determine if the new measurement is anomalous (EPA 2010). The development and deployment of a sub-zoning software tool is used to help to simplify the distribution network structure by organizing the water consumers in (virtual) sub-zones/clusters. This simplified tree-like cluster graph will improve the understanding of how water quality parameters such as total residual chlorine levels and water age are affected by the network topology.

\section{PUB experience with technology \& gaps identified}

Besides leak detection, the WaterWise system also includes multi-parameter probes that enhance WSN's ability to detect water quality issues in real-time. Each multisensor probe measures the following water quality parameters: $\mathrm{pH}$, ORP, conductivity, temperature and turbidity. Coupled with an event detection system to identify anomalous events, the real-time acquisition of water quality data helps us strengthen water security and enhance incident management. WSN is continually exploring the use of other sensors and indicators to either detect a broader spectrum of contaminants in the network and/or provide an earlier warning system. The CANARY event detection system has been installed in WSN's control centre. However, it is challenging to validate sensors and data analytics tools in a "live" network. Dramatic changes in water quality parameter values can result from a variety of benign causes such as changes in water demands, system operations, and source water variability. Furthermore, inaccurate data may be received due to sensor or data communication issues. At present, WSN is integrating CANARY into WaterWise and adapting CANARY to better suit operational requirements.

WSN has tested Optiqua's generic Eventlab sensor (http://www.optiqua.com/eventlab. html), which measures changes in refractive index of water to detect any water quality variations due to dissolved substances (Optiqua 2014; Raich 2013). However, the Eventlab sensors cannot work in isolation, as they are unable to detect undissolved particles and biological agents due to the 0.5 or 1.0 micron size filter that is installed as part of the system. WSN uses live tiger barbs as a biosensor for real-time monitoring of contaminants. Fish monitoring systems have been deployed for the monitoring of treated water at service reservoirs. The Fish Activity Monitoring System, developed by PUB and the Institute of 
Infocomm Research $\left(\mathrm{I}^{2} \mathrm{R}\right)$ (http://www.i2r.a-star.edu.sg/), allows the control centre to monitor the tiger barbs remotely, with video analytics providing automated alerts when the number of fish deaths exceeds a threshold (Eng et al. 2008).

\section{Technology roadmap}

The goal for water quality management in the distribution network is to fully implement real-time sensing methods and data analytical tools in the network operations to achieve real-time monitoring and modelling of water quality in the entire water distribution system. WSN targets to deploy more than 300 sensors (including feasible routine sampling points) by 2017, covering a wide range of the contaminant spectrum. With the large amount of sensor data collected, WSN will carry out data mining to improve on the accuracy and usability of event detection, source-tracing and subzoning algorithms. WSN aims to own a real-time water quality model by 2030 . Such a model will help to determine areas that may require additional interventions, such as flushing and/or additional disinfection.

\section{Automated meter reading}

\section{Objectives \& challenges}

WSN currently takes charge of more than 1.4 million mechanical water meters for billing customers. SP Services Limited (SPS) (http://www.singaporepower.com.sg/irj/ portal/spservices) handles accounts and billing matters on our behalf. Water, electricity and gas meters are read manually by SPS meter readers on a bimonthly basis. Unfortunately, the low frequency of manual meter readings does not offer sufficient granularity for understanding customer water consumption patterns. With automated meter reading (AMR) systems in place, besides having actual meter readings for monthly billing, WSN will be able to provide customers with hourly consumption data for them to better understand and control their water usage, and even identify suspected water leaks. AMR data can also be used for water consumption behaviour analysis to develop targeted water conservation measures. Aggregated AMR data, together with other smart sensor information, could also be useful for network system performance analysis such as water balance studies. The long term objective for AMR is to achieve an accurate, low cost AMR system suitable for meter reading and collecting useful data for network optimization as well as water conservation efforts.

\section{Review of current technology \& tools in the market}

A fixed network AMR system will be suitable for providing granular data for the purposes of water conservation and network optimization. Figure 2 shows a schematic diagram of a typical AMR system. The key considerations in selecting an AMR system are reliable endpoints (meter interface unit) and its mode of communication to the data concentrator. Singapore is highly urbanized, which creates a challenge for fixed network AMR deployment, because the design of the radio network has to consider presence of physical obstacles to RF propagation.

Through pilot trials, WSN has tested various fixed network AMR technologies. These systems include short range systems with endpoints operating in unlicensed free Radio Frequency (RF) bands (UHF) and their data concentrators using cellular 2G/3G networks. WSN has also tested some systems with endpoints directly using 2G/3G 


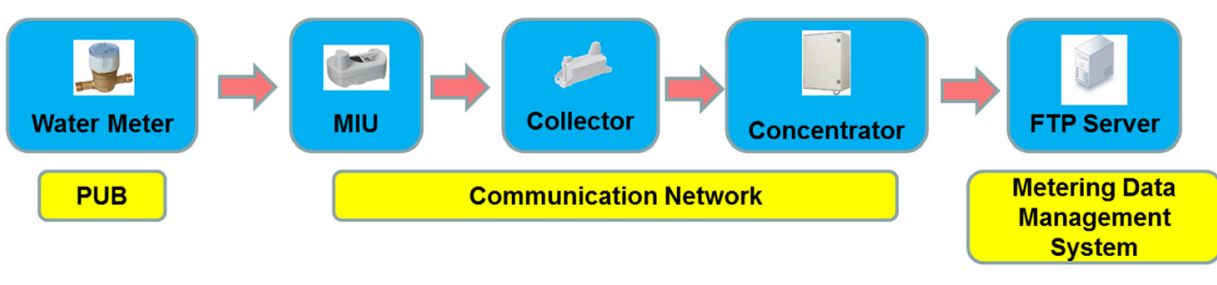

Fig. 2 Typical schematic of fixed network AMR system

networks. Moving forward, WSN is also testing long range systems with endpoints operating in unlicensed free RF bands (VHF) as well as shared networks using open communication standards. While fixed AMR systems can vary in design in terms of configuration and RF propagation, ongoing trials show that most systems perform well in terms of availability, given that site and environment considerations are taken care of during system design. WSN is evaluating the performance and overall costs of different systems as well as the complexity and reliability in operation. WSN is currently exploring open communication standards to allow flexibility in deploying meter and network components in the future. If we can leverage on existing or future shared networks, this could lower the costs in setup and operation of data communication infrastructure. Due to the high ambient temperature and humidity in Singapore, AMR components, such as endpoints, need to be sealed against water and designed to work reliably in hot environments. Trials show that fully potted designs tend to perform better in preventing moisture ingress which can damage electronic parts. The endpoints for water must be battery powered and battery life should be at least equivalent to the lifecycle of the meter. Battery life will be considerably shortened when operating under high temperature, especially those exposed under direct sunlight.

\section{PUB experience with technology \& gaps identified}

WSN had previously piloted two fixed network AMR systems in 2010 to assess the technical feasibility of deploying short-range fixed network AMR systems in Singapore's high-rise residential estates (Toa Payoh, Punggol) and low-rise industrial estates (Tuas Industrial Estate). WSN had also successfully implemented AMR to acquire water consumption data from the top 100 commercial and industrial customers in 2014. The data from the first phase (Top 100) provided a good sensing of the causes behind the fluctuations of Singapore's daily demand, especially in analysing the increase in Singapore's demand during the dry season. In the next phase, WSN will extend AMR to the Top 600 customers by Q1 2016. The implementation costs of AMR remains high and WSN is exploring ways to lower these costs. The two key measures of AMR reliability are accuracy and reading availability and WSN is testing systems to evaluate this.

\section{Technology roadmap}

The ideal AMR system will be a system of low complexity and low power to provide a stable, reliable and secure data exchange. To tackle the costs of deploying AMR, WSN is exploring the use of shared infrastructure networks, and methods to eliminate the need to replace existing meters. WSN is working with the Infocomm Development Authority of Singapore (IDA) under the Smart Nation Platform (SNP) to explore the 
feasibility of reading water meters using a low-power wireless sensor shared network in Yuhua. WSN is also working with $\mathrm{I}^{2} \mathrm{R}$ to develop a low-power image recognition device with short-range low power wireless communication. Optical character recognition (OCR) and image processing technologies will be used to provide accurate meter readings, while also reducing the need to replace meters for AMR as the device can be easily installed on existing meters. WSN has embarked on a project with Suez Environnement (http://www.suez-environnement.com/) to study how to induce behavioural change towards water conservation using Advanced Metering Infrastructure (AMI), data analytics and customer engagement. Suez will install 1200 AMRs to collect data for analytics and use customer engagement to influence customer water usage behaviour. Out of these 1200 AMRs, 600 will be installed in Yuhua in conjunction with the SNP. The remaining 600 AMRs will be installed in Punggol using the Suez VHF system. Apart from assessing the feasibility and reliability of AMR, Suez will demonstrate the value of AMR data in water conservation and network operations. Separately, WSN is also exploring with Singapore Power PowerGrid (SPPG) (http://www.singaporepower. com.sg/irj/portal) to leverage on their pervasive electricity AMI to read water meters. SPPG is using their existing proprietary AMI system for billing electricity contestable customers. WSN targets to identify feasible AMR solutions by 2018 and roll-out AMR to all customer premises by 2028 .

\section{Water conservation}

\section{Objectives \& challenges}

Studies based on behavioural economics and psychology show that providing users with real time information can lead to changes in behaviour. In order to achieve the target of 147 litres/capita/day by 2020 and 140 litres/capita/day by 2030, WSN is exploring how real time information and feedback from smart water-efficient gadgets/fittings \& technologies could drive water consumption behavioural changes of domestic customers. Trust and data protection is important as water consumption data could be sensitive and personal. In addition, cost effectiveness is a major consideration for any home water management solutions and it is often challenging to justify the cost effectiveness of smart water-efficient gadgets.

\section{Review of current technology \& tools in the market}

In a typical household, showers consume the most water (29\% of an average family's monthly water consumption, (Public Utilities Board 2016)). Encouraging further flow rate reduction in shower mixers usually leads to a loss of comfort. Emerging water-efficient showerheads utilize technology that can maintain or increase comfort levels even at low flow rates (e.g. air intake, pulsating flow, and automatic reduction of flow strength based on proximity to user). Some showerheads make use of hydromotors with LED lights that change colour according to the shower duration. Finally, there are also devices which can be installed to showerheads to provide real time feedback on water and energy usage to consumers. The Amphiro (http://www.amphiro.com/) device is one example of such a device that is also integrated with an online portal that displays usage trends. While home energy management solutions are already widely available in the market, home water management solutions are still uncommon. The current solutions are able to 
provide the total water consumption data to the home owners with the help of smart water metering, at intervals as short as $10 \mathrm{~s}$. Technologies are also available to give more intricate information on water uses at homes. Flow-trace analysis software such as Trace Wizard disaggregates residential water use into its component end uses, providing a detailed breakdown of the residential water usage to home dwellers (Wong et al. 2012).

\section{PUB experience with technology \& gaps identified}

WSN has collaborated with Rigel Technology(s) Pte Ltd (http://www.rigel.com.sg/) to develop showerhead prototypes which are equipped with LED lights and consumption displays to indicate the duration of shower and amount of water consumed to the user. $50 \%$ of users who tested the showerhead prototype found their consumption dropped by an average of $9.6 \%$. However, the study noted the importance of good design to ensure that the commercialized version is appealing to the mass market. Under the Smart Water Meter project, WSN has performed a trial to evaluate the feasibility of disaggregating residential water use by using a flow trace analysis software, Trace Wizard. However, more research is needed to enhance the accuracy especially for events with similar flow patterns. There is also a lack of processing capabilities such as the analysis of multi-cycle shower patterns and simultaneous water uses. There is also a trade-off between battery life and data resolution for battery operated data-logging and/or transmission devices mounted on smart water meters. For instance, the Trace Wizard software (http:// www.aquacraft.com/downloads/trace-wizard-description/) requires flow data which are at $10 \mathrm{~s}$ intervals, but this will cause the battery life of the Meter Interface Units to deplete in a few months. On the other hand, lower resolution data might not be able to produce meaningful analytical results.

\section{Technology roadmap}

Future development in water demand management technologies will be focused on inducing behavioural change by improving the provision of real-time information and analysis on water consumption behaviour. Interactive engagement analytical tools will be developed, leveraging on well-established behavioural change strategies like gamification in order to encourage water conservation among PUB's customers. Under the Smart Nation initiative, WSN has embarked on a field trial of smart-enabled homes (see Fig. 3) with home water management systems available to 3200 households in the Yuhua estate (Loh 2016). By 2017, the outcomes from the trial will be used to further behavioural studies to evaluate the effectiveness of providing water consumption information to customers.

\section{Conclusion}

The implementation of a Smart Water Grid system supports WSN's mission to supply good water $24 / 7$ to its customers. With sensors and analytic tools deployed island-wide to provide a real-time monitoring and decision support system, the Smart Water Grid system enables WSN to manage the water supply network efficiently, ensuring that all Singaporeans will continue to enjoy a reliable and sustainable water supply for generations to come. 


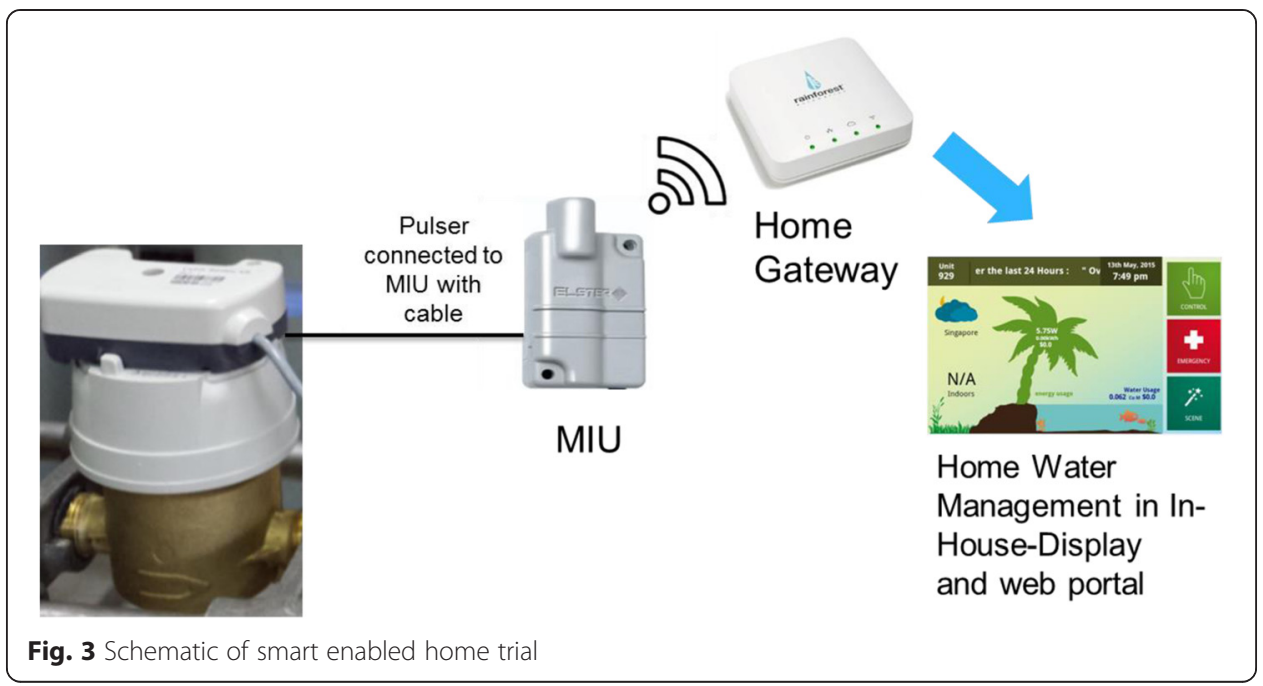

This paper has highlighted WSN's vision for the five key aspects of the Smart Water Grid system, in relation to both the network system (asset management, leak management, water quality monitoring) and the customers' end (automated meter reading, and water conservation). With improved asset management, effective preventive maintenance of high-risk pipes will further reduce the number of leaks and water quality incidents. In tandem, as the networks of real-time pressure and water quality sensors continue to be expanded, WSN will also be equipped with the necessary data and tools for timely incident management. At the customers' end, real-time water consumption information from the automated meter readings will help customers to make informed choices towards water conservation within their homes and company premises.

The technology development roadmap for each aspect of the Smart Water Grid system follows a common theme: the adoption of real-time sensing methods and data analytical tools as ICT and analytical software solutions mature and new technologies emerge to better meet the needs of water utilities and customers. PUB currently sets aside an annual budget of $\$ 20$ million for R\&D ranging from upstream research to demonstration-scale projects. Funding is also available from the TechPioneer scheme administered by Singapore's Economic Development Board, which encourages companies to accelerate the commercialisation of new water technologies by encouraging their early adoption in Singapore. WSN will continue to experiment, test-bed and work with industrial and academic partners to accelerate the adoption of these emerging technologies to better support the implementation of the Smart Water Grid in Singapore's context.

Abbreviations

AMI, advanced metering infrastructure; AMR, automated meter reading; COF, consequence of failure; ICT, information and communications technologies; IDA, Infocomm Development Authority of Singapore; I2R, Institute of Infocomm Research; LOF, likelihood of failure; MGD, millions of gallons per day; OCR, optical character recognition; ORP, oxidation-reduction potential; PUB, Public Utilities Board; RF, radio frequency; SNP, Smart Nation Platform; SPPG, Singapore Power PowerGrid; SPS, SP Services Limited; UHF, ultra high frequency; VHF, very high frequency; WSN, Water Supply Network Department 


\section{Acknowledgements}

Editors:

Lai Kah Cheong, Ong Kok Hsing, Pan Ju Khuan

Chapter authors:

Asset Management: Varatharajan Ramaswamy

Leak Management: Ong Kok Hsing

Water Quality Monitoring: Irene Toh \& Sarah Teng

Automated Meter Reading: Soh Yake Leong

Water Conservation: Wong Waicheng

With inputs from:

Jaslyn Goh, Sheryl Chia, Lim Wee Beng, Qiu Tian, Li Qiming, Kelvin Loh, Joann Lim, Cheng Zesen, Stanley Woo,

Peng Kaiqi, Li Mengmeng, Erin Seow, Kuy Wah Leong, Tan Kah Kyee, Gayathri Kalyanaraman, Amelia Koh, Adeline Neo,

Shen Haixi, Tan Boon Lin, Yuen Yu Leng, Edwin Chin, Matthew Loh, Wong Yueat Tin, Zhou Kaijing

In consultation with:

Michael Toh Director (Water Supply Network)

Chong Hou Chun Senior Deputy Director (Water Supply Network)

$\mathrm{Ng}$ Han Tong Senior Deputy Director (Water Supply Network)

Wong Kee Wei Chief Specialist (Water Supply Network)

Lim Mong Hoo Chief Specialist (Water Quality)

Harry Seah Chief Engineering \& Technology Officer

\section{Funding}

No funding was received for the preparation of this review.

\section{Availability of data and materials}

NA.

\section{Competing interests}

The authors declare that they have no competing interests.

Received: 9 June 2016 Accepted: 15 June 2016

Published online: 21 July 2016

\section{References}

Allen M, Preis A, lobal M, Srirangarajan S, Lim HB, Girod L, Whittle AJ (2011) Real-time in-network distribution system monitoring to improve operational efficiency. Am Water Works Assoc J 103(7):63

Eng HL, Lee BH, Lim E, Anggrelly SY, Chew BF, Tai YC, Quek J, Haja N, Teo CP and Wong YT (2008) A Fish Activity Monitoring System for Early Detection of Water Contamination. A*STAR Open Access Repository. http://oar.a-star.edu.sg:80/jspui/handle/123456789/1283. Accessed 16 Feb 2016.

Hamilton S, Charalambous B (2013) Leak detection: Technology and Implementation. 1st ed. IWA Publishing, London, UK

Loh CJ (2016) Smart devices trial extended to 3,200 households in Yuhua. Channel News Asia. Retrieved from http://www.channelnewsasia.com/. Accessed 16 Feb 2016

Optiqua Technologies (2014) The benefits of using refractive index for water quality monitoring in distribution networks. http://www.optiqua.com/documents/20140804_OptiquaEventLabcomparison_withcover.pdf. Accessed on 16 Nov 2015

Pradhan T, Aslam S, Chia S (2014) Predicting potential water pipe failures ahead of time. Innov Water 6:22, https:/www.pub.gov.sg/Documents_Nol_6.pdf. Accessed 16 Feb 2016.

Public Utilities Board (2016) Water Saving Habits. https:/www.pub.gov.sg/savewater/athome/watersavinghabits. Accessed on 16 Feb 2016

Raich J (2013) Review of sensors to monitor water quality. European Reference Network for Critical Infrastructure Protection (ERNCIP) project

U.S. EPA (2010) Water Quality Event Detection Systems For Drinking Water Contamination Warning Systems: Development, Testing, and Application of CANARY. https://cfpub.epa.gov/si/si_public_record_report. cfm?dirEntryld=221394. Accessed on 16 Nov 2015

Whittle A, Girod L, Preis A, Allen M, Lim H, Iqbal M, Srirangarajan S, Fu C, Wong K, Goldsmith D (2011) WaterWiSe@SG: A Testbed for Continuous Monitoring of the Water Distribution System in Singapore. Water Distribution Systems

Wong WC, Kok TW, Putri N, Kalyanaraman G, Chin E, Lee KS (2012) Getting smarter about residential water. Innov Water 3:22, https://www.pub.gov.sg/Documents/InnovationWater_vol3.pdf. Accessed 16 Feb 2016. 